\title{
Dose-dependent impact of larval Ascaris suum on host body weight in the mouse model
}

\author{
R. Lewis ${ }^{1}$, J.M. Behnke ${ }^{2}$, P. Stafford ${ }^{1}$ and C.V. Holland ${ }^{1 *}$ \\ ${ }^{1}$ Department of Zoology, School of Natural Sciences, Trinity College, \\ University of Dublin, Dublin 2, Ireland: ${ }^{2}$ School of Biology, University of \\ Nottingham, University Park, Nottingham NG7 2RD, UK
}

\begin{abstract}
Ascaris lumbricoides and Ascaris suum are important helminth parasites of humans and pigs, respectively. Although it is now well established that the presence of mature adult worms in the host intestine contributes to significant nutritional morbidity, the impact of larval migratory ascariasis is far less well understood. The development of a mouse model to explore susceptibility and resistance to larval ascariasis in the lungs provided an opportunity to observe the impact of larval migration on host growth during the course of infection. Changes in body weight were monitored in two strains of inbred mice, the susceptible C57BL/6j and the resistant CBA/Ca. Groups of mice received one of four doses: 100, 500, 1000 and 3000 fully embryonated $A$. suum ova. Infected mice underwent post-mortem on days 6, 7 and 8 post-infection. Control mice received a placebo dose of intubation medium and underwent post-mortem on day 7 post-infection. Mice were weighed pre-infection (day 0) and post-infection on the day of post-mortem. At post-mortem, the lungs of each mouse were removed for enumeration of Ascaris larval burdens by means of the modified Baermann method. Control mice of each strain showed an increase in weight from preinfection to post-infection day. Within the C57BL/6j strain, mice infected with higher doses of Ascaris eggs experienced a reduction in body weight; for those given 3000 eggs this was on all three post-mortem days, and for those given 1000, on days 7 and 8. For CBA/Ca mice, only mice receiving the 3000 dose demonstrated a reduction in body weight. These findings suggest that larval migratory ascariasis has a significant negative impact upon host growth and that this is related to infective dose and larval burden.
\end{abstract}

\section{Introduction}

Ascaris lumbricoides and Ascaris suum are important helminth parasites of humans and pigs, respectively (Roepstorff \& Nansen, 1994; Holland, 2005). A. lumbricoides is believed to infect approximately 1472 million people worldwide and to contribute to an estimated 11.81 million thousand disability-adjusted life years (DALYS) (Chan, 1997; WHO, 2002).

*Fax: 353-1-6778094

E-mail: cholland@tcd.ie
The major impact of these infections is chronic nutritional impairment, although more acute morbidity, including intestinal obstruction, has been documented (Crompton, 2001). It is now well established that the presence of mature adult worms in the host intestine contributes to reduced food intake, impaired digestion, malabsorption and poor growth rates (reviewed in Crompton \& Nesheim, 2002). These observations have been made in both experimental infections in pigs (Stephenson et al., 1980) and field-based studies in children (Hlaing, 1993; O'Lorcain \& Holland, 2000). The extent of nutritional impairment is related to the intensity of infection (O'Lorcain \& Holland, 2000). 
However, the impact of larval migratory ascariasis on its host is far less well understood (Cooper et al., 1992). As outlined by Stephenson (1987), the difficulty of designing appropriate and ethical studies of larval migration in humans has impeded research into this phase of the life cycle and, consequently, the public health significance remains unclear. Larval migration can cause pneumonitis, including asthma, cough, substernal pain, fever, skin rash and eosinophilia (Coles, 1985; Pawlowski \& Arfaa, 1985). These symptoms have been postulated to contribute to reduced food intake and, in the case of fever, increased nitrogen loss (Stephenson, 1987). In the rat, infection by Nippostrongylus brasiliensis, another intestinal nematode which undergoes lung migration, induces a biphasic anorexia, the first phase associated with lung invasion, the second when worms mature in the intestine (Ovington, 1985; Mercer et al., 2000).

The development of a mouse model to explore susceptibility and resistance to larval ascariasis in the lungs (Lewis et al., 2006, 2007) provided an opportunity to observe the impact of larval migration on host growth during the course of infection. In this paper, we describe the changes in body weight observed in two strains of inbred mice, the susceptible C57BL/6j and the resistant $\mathrm{CBA} / \mathrm{Ca}$, infected with low, medium and high doses of Ascaris eggs.

\section{Materials and methods}

\section{Experimental animals}

One hundred and twenty inbred male mice, 60 C57BL/6j and $60 \mathrm{CBA} / \mathrm{Ca}$ were purchased from Harlan, UK at 7 weeks of age and were 8 weeks old on the day of infection. Animals were maintained under standard and constant conditions, as described by Lewis et al. (2006).

\section{Parasite}

Approximately 4,000,000 embryonated ova (batch number: 8/2002) were provided by the Danish Centre for Experimental Parasitology (CEP), Copenhagen. Mice were inoculated by gastric intubation, between 08.00 and 10.00 hours, with doses of fully embryonated eggs, as described in Lewis et al. (2006).

\section{Experimental design and larval recovery}

Fifteen mice of each strain were inoculated with one of four single-inoculum doses (100, 500, 1000 and 3000). Ten mice of each strain received a placebo dose consisting of intubation medium only. Infected mice (five mice per dose) underwent post-mortem examination on days 6, 7 and 8 post-infection. In Ascaris-infected mice, the maximum accumulation of larvae by the susceptible strain is consistently observed in the lungs on day 7 postinfection and as such, this day is suggested to be the optimum for assessing differences in susceptibility (Lewis et al., 2006). Control mice underwent post-mortem examination on day 7 . These age-matched controls were maintained separately from infected mice at all times during the experiment. All mice were euthanized by cervical dislocation on the relevant post-mortem day.

Mice were weighed pre-infection (day 0) and postinfection on the day of post-mortem. At post-mortem, the lungs of each mouse were removed for enumeration of Ascaris larval burdens by means of the modified Baermann method (Lewis et al., 2006). Larval counts were performed on five, $200 \mu \mathrm{l}$ samples taken from the product of centrifugation. These experiments were reviewed and approved by the university research ethics committee and the Department of Health and Children (Ireland).

\section{Statistical analysis}

Mouse body weight was analysed separately for each strain, as inherent strain differences might have influenced the statistical analysis. Therefore within each strain, the influence of dose of infection and day of post-mortem on the difference in host body weight (between preinfection and the day of post-mortem) was explored by means of two-way analysis of variance (ANOVA). Least squares difference (LSD) post-hoc tests were applied to compare differences within the factor of dose. Statistical analysis was carried out at a confidence limit of $95 \%$ $(\alpha=0.05)$.

\section{Results}

Larval numbers of A. suum in the lungs of susceptible and resistant strains of mice

The numbers of larvae recovered from the lungs of mice of each strain at each dose and on each day of postmortem are shown in table 1. As discussed previously, there was a highly significant positive relationship between dose of eggs administered and the larval burdens, with strain and day taken into account, suggesting a close correlation between the administered dose size and the number of larvae recovered within each strain (Lewis et al., 2006).

\section{Impact of infection with A. suum on host body weight}

Control mice of both strains showed an increase in weight of $1 \mathrm{~g}$ from pre-infection to post-infection day (see table 2). In contrast, some groups of infected mice demonstrated reductions in body weight after infection with Ascaris eggs and during larval migration in the lungs.

Within the C57BL/6j strain, mice infected with higher doses of Ascaris eggs experienced a reduction in body weight; for those given 3000 eggs this was on all three post-mortem days, and for those given 1000 eggs, on days 7 and 8 (fig. 1a). Statistical analysis revealed that dose had a significant influence on body weight (twoway ANOVA with dose and day as factors, model $R_{\text {adj }}^{2}=0.85$, main effect of dose, $F_{3,48}=37.2, P \leq 0.0001$ ). In contrast, mice infected with the lower doses generally showed an overall increase in weight. This was also reflected in the post-hoc analysis for the C57BL/6j strain, which revealed that the 3000 and 1000 dosage groups differed significantly from each other in terms of 
Impact of larval Ascaris suum on mouse body weight

Table 1. A comparison of the total number of worms recovered ( \pm SEM) from the lung in both mouse strains (C57BL/6j and CBA/Ca) for each of the single-pulse infections $(3000,1000,500$ and $100 \mathrm{~A}$. suum ova) on the three post-mortem days (days 6-8).

\begin{tabular}{lcccc}
\hline Strain & Day & $n$ & Dose & Lung total \\
\hline C57BL/6j & 6 & 5 & 3000 & $456.8 \pm 106.4$ \\
& 7 & 5 & & $647.2 \pm 108.4$ \\
& 8 & 5 & & $422.2 \pm 60.1$ \\
& 6 & 5 & 1000 & $76 \pm 18.0$ \\
& 7 & 5 & & $164 \pm 21.6$ \\
& 8 & 5 & & $82.4 \pm 22.2$ \\
& 6 & 5 & 500 & $44.0 \pm 5.7$ \\
& 7 & 5 & & $60.8 \pm 17.3$ \\
& 8 & 5 & & $30.4 \pm 5.6$ \\
& 6 & 5 & 100 & $4.8 \pm 3.2$ \\
& 7 & 5 & & $7.2 \pm 3.2$ \\
& 8 & 5 & & $6.4 \pm 2.4$ \\
& 6 & 5 & 3000 & $252.0 \pm 33.9$ \\
& 7 & 5 & & $215.2 \pm 30.3$ \\
& 8 & 5 & & $187.2 \pm 9.6$ \\
& 6 & 5 & 1000 & $32.8 \pm 6.6$ \\
& 7 & 5 & & $38.4 \pm 8.5$ \\
& 8 & 5 & & $32.0 \pm 4.2$ \\
& 6 & 5 & 500 & $12.8 \pm 3.6$ \\
& 7 & 5 & & $9.6 \pm 2.9$ \\
& 8 & 5 & & 0 \\
6 & 4 & 100 & $2.4 \pm 1.6$ \\
& 7 & 5 & & $0.8 \pm 0.8$ \\
\hline & 8 & 5 & &
\end{tabular}

changes in body weight $(P \leq 0.0001)$. These higher-dose groups also differed significantly $(P \leq 0.0001)$ from the lower-dose groups, which did not differ from each other. There was also a significant effect of days postinfection $\left(F_{2,48}=22.8, P \leq 0.0001\right)$ and the interaction between dose and days post-infection $\left(F_{6,47}=3.0\right.$, $P \leq 0.0126$ ). With the exception of the 100 dose group, body weight declined from day 6 to day 8 .

At all dose levels, CBA/Ca mice had significantly fewer larvae in their lungs compared to C57BL/6j (table 1). Statistical analysis did reveal a significant effect of dose on mouse body weight (two-way ANOVA with dose and day as factors, model $R_{\text {adj }}^{2}=0.699$, main effect of dose, $F_{3,47}=27.3, P \leq 0.0001$ ) with only mice receiving the 3000 egg dose demonstrating a reduction in body weight (fig. 1b). Post-hoc analysis revealed that the loss of body weight in mice receiving the highest dose was significantly different from all other groups $(P \leq 0.0001)$ which did not differ from each other. There was also a significant effect of days post infection $\left(F_{2,47}=9.8, P \leq 0.0001\right)$ and the interaction between dose and days post infection $\left(F_{6,47}=7.3, P \leq 0.0001\right)$. In contrast to the C57BL/6j mice, the effect of days post infection in CBA/Ca mice was inconsistent, with weights fluctuating up and down during the period of infection.

\section{Discussion}

This experiment provides evidence that the migration and accumulation of $A$. suum larvae in the lungs of mice has a significant impact upon host fitness and,
Table 2. Mean body weight $(\mathrm{g})( \pm \mathrm{SEM})$ at pre-infection (time zero) and on each post-mortem day of groups of both C57BL/6j and $\mathrm{CBA} / \mathrm{Ca}$ mouse strains treated with different doses of A. suum ova. Also shown are the weights of the day 7 control groups for both strains.

\begin{tabular}{|c|c|c|c|c|c|}
\hline Strain & Dose & Day & $n$ & $\begin{array}{c}\text { Pre-infection } \\
\text { weight } \\
( \pm \text { SEM })\end{array}$ & $\begin{array}{l}\text { Post-infection } \\
\text { weight } \\
( \pm \text { SEM })\end{array}$ \\
\hline \multirow[t]{13}{*}{ C57BL/6j } & \multirow[t]{3}{*}{3000} & 6 & 5 & $15.84 \pm 0.64$ & $15.30 \pm 0.57$ \\
\hline & & 7 & 5 & $16.34 \pm 0.46$ & $14.40 \pm 0.19$ \\
\hline & & 8 & 5 & $15.42 \pm 0.62$ & $12.68 \pm 0.61$ \\
\hline & \multirow[t]{3}{*}{1000} & 6 & 5 & $16.46 \pm 0.26$ & $17.20 \pm 0.08$ \\
\hline & & 7 & 5 & $15.92 \pm 0.52$ & $15.28 \pm 0.24$ \\
\hline & & 8 & 5 & $16.02 \pm 0.51$ & $14.34 \pm 0.07$ \\
\hline & \multirow[t]{3}{*}{500} & 6 & 5 & $16.72 \pm 0.23$ & $17.64 \pm 0.27$ \\
\hline & & 7 & 5 & $17.06 \pm 0.51$ & $17.54 \pm 0.39$ \\
\hline & & 8 & 5 & $16.40 \pm 0.37$ & $16.38 \pm 0.33$ \\
\hline & \multirow[t]{3}{*}{100} & 6 & 5 & $16.06 \pm 0.54$ & $16.78 \pm 0.61$ \\
\hline & & 7 & 5 & $16.10 \pm 0.62$ & $16.52 \pm 0.61$ \\
\hline & & 8 & 5 & $16.54 \pm 0.36$ & $17.06 \pm 0.28$ \\
\hline & Control & 7 & 10 & $19.8 \pm 0.50$ & $20.8 \pm 0.39$ \\
\hline \multirow[t]{13}{*}{$\mathrm{CBA} / \mathrm{Ca}$} & \multirow[t]{3}{*}{3000} & 6 & 5 & $20.54 \pm 0.90$ & $20.94 \pm 0.77$ \\
\hline & & 7 & 5 & $21.10 \pm 0.62$ & $20.02 \pm 0.56$ \\
\hline & & 8 & 5 & $20.80 \pm 0.51$ & $18.04 \pm 0.45$ \\
\hline & \multirow{3}{*}{1000} & 6 & 5 & $20.32 \pm 0.28$ & $20.84 \pm 0.32$ \\
\hline & & 7 & 5 & $20.16 \pm 0.34$ & $21.04 \pm 0.28$ \\
\hline & & 8 & 5 & $20.22 \pm 0.29$ & $21.00 \pm 0.35$ \\
\hline & \multirow[t]{3}{*}{500} & 6 & 5 & $22.42 \pm 0.79$ & $23.24 \pm 0.83$ \\
\hline & & 7 & 5 & $22.96 \pm 0.51$ & $23.52 \pm 0.49$ \\
\hline & & 8 & 5 & $21.82 \pm 1.02$ & $22.22 \pm 0.92$ \\
\hline & \multirow[t]{3}{*}{100} & 6 & 4 & $23.58 \pm 0.70$ & $24.00 \pm 0.87$ \\
\hline & & 7 & 5 & $21.54 \pm 1.15$ & $21.80 \pm 0.97$ \\
\hline & & 8 & 5 & $22.72 \pm 0.38$ & $22.86 \pm 0.34$ \\
\hline & Control & 7 & 10 & $19.8 \pm 0.33$ & $20.8 \pm 0.36$ \\
\hline
\end{tabular}

specifically, host body weight. This effect is most pronounced at higher infection levels after a dose of 3000 eggs has been administered $(12 \%$ and $17.8 \%$ body weight loss in C57BL/6j mice at 7 and 8 days postinfection). It is of interest to note that, albeit to a lesser extent, larval ascariasis also had an impact on the resistant CBA/Ca strain of mice, particularly on day 8 of infection (13.3\% body weight loss), in those mice that received a dose of 3000 eggs. In contrast, control animals of both strains gained weight over the same time period ( $5 \%$ body weight gain), as did some infected mice that received lower-dose infections.

Currently, evidence from both human and porcine studies points to the presence of adult worms in the intestine contributing to nutritional impairment, including growth retardation (Stephenson et al., 1980; Hale et al., 1985; child growth studies reviewed in O'Lorcain \& Holland, 2000). Furthermore, after deworming, infected children show improvements in growth and appetite (Hadju et al., 1996).

In contrast, there is a paucity of data on the impact of larval ascariasis on host fitness, including nutritional status and body weight. Part of the explanation for this relates to the difficulty of establishing a causal relationship between larval migration and pathogenesis under field conditions. Hale et al. (1985) concluded that the effect of migrating larvae was less pronounced than that of adult worms, and Stephenson et al. (1980) infected pigs 

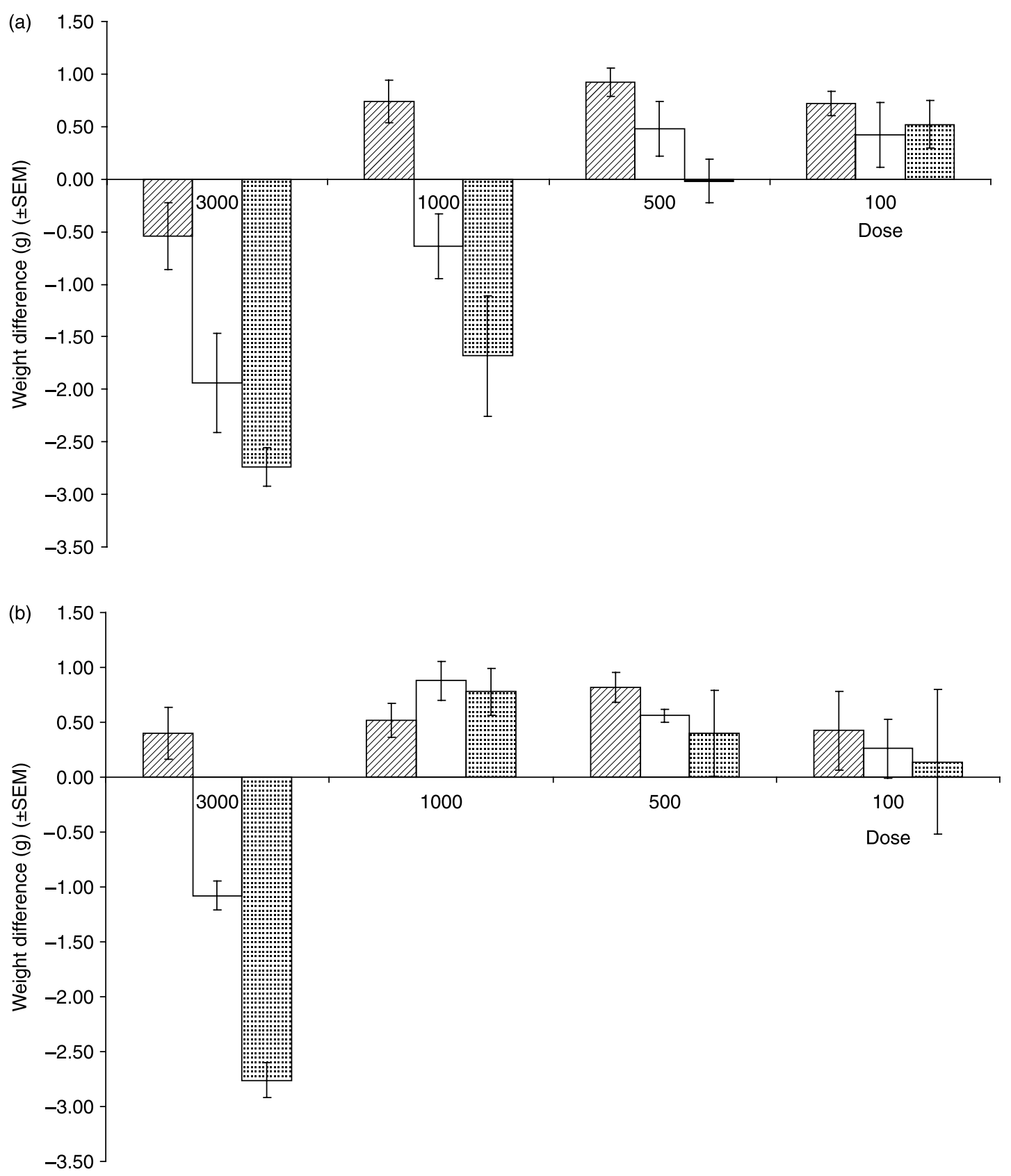

Fig. 1. Mean body weight difference $(g)( \pm$ SEM) between pre-infection (time zero) and the day of post-mortem $( \pm$ SEM) in both the (a) C57BL/6j mouse strain and (b) CBA/Ca mouse strain, on each post-mortem day (घ, Day 6; $\square$, Day 7; 田, Day 8).

with fourth-stage larvae and demonstrated that adult worms depressed the growth rate in the absence of larval migration. Nevertheless, fenbendazole treatment during the liver migration phase improved feed conversion ratios by $22 \%$, and treatment during pulmonary migration improved these rates by $8 \%$ in comparison to controls (Stewart et al., 1984). The underlying mechanisms resulting in these reduced growth/feed ratios in pigs may be due to parasite-induced physiological changes.
Yang et al. (1990) observed a reduced gastrin level during the lung migration phase and increased cholecystokinin (CCK) levels after larvae had reached the pig intestine.

In a different species, rats infected with the rodent nematode $N$. brasiliensis consumed dramatically less food early in infection (on day 2 after infection, which corresponds to the arrival and duration of larval stages in the lungs) (Ovington, 1985). Furthermore, the extent of reduced food intake was related to the dose of 
$N$. brasiliensis larvae administered, when the range of doses was expressed as numbers of larvae per gram of body weight.

The current examination of weight changes and Ascaris larval burden in two inbred strains of mice highlights the impact of host susceptibility and infective dose on host fitness. Animals with similar larval burdens, irrespective of strain, displayed analogous weight changes during the period of observation. This suggests that there is a negative impact on host fitness in relation to larval ascariasis and that this relates to larval burden and exposure to larval ascariasis, rather than primarily to host genetic status and associated host susceptibility/resistance. It also highlights the potential negative impact of nematode migration upon growth, which is most likely mediated by a reduction in food intake.

\section{Acknowledgements}

We thank the Irish Research Council for Science, Engineering and Technology and the EMBARK initiative for funding the research, and Professor Lis Eriksen in the Royal Veterinary College, Copenhagen for the provision of A. suum eggs and her help and support. We would also like to thank Clare Hamilton, Alison Boyce and Brian Murphy for their hard work in the laboratory.

\section{References}

Chan, M.S. (1997) The global burden of intestinal nematode infection - fifty years on. Parasitology Today 13, 438-443.

Coles, G.C. (1985) Allergy and immunopathology of ascariasis. pp. 167-184 in Crompton, D.W.T., Nesheim, M.C. \& Pawlowski, Z.S. (Eds) Ascariasis and its public health significance. London: Taylor \& Francis.

Cooper, E.S., Whyte-Alleng, C.A.M., Finzi-Smith, J.S. \& MacDonald, T.T. (1992) Intestinal nematode infections in children: the pathophysiological price paid. Parasitology 104, S91-S103.

Crompton, D.W.T. (2001) Ascaris and ascariasis. Advances in Parasitology 48, 285-374.

Crompton, D.W.T. \& Nesheim, M.C. (2002) Nutritional impact of intestinal helminthiasis during the human life cycle. Annual Reviews in Nutrition 22, 35-59.

Hadju, V., Stephenson, L.S., Abadi, K., Mohammed, H.O., Bowman, D.D. \& Parker, R.S. (1996) Improvements in appetite and growth in helminth-infected schoolboys three and seven weeks after a single dose of pyrantel pamoate. Parasitology 113, 497-504.

Hale, O.M., Stewart, T.B. \& Marti, O.G. (1985) Influence of experimental infection of Ascaris suum on performance in pigs. Journal of Animal Science 60, 220-225.

Holland, C.V. (2005) Gastrointestinal nematodes Ascaris, hookworm, Trichuris and Enterobius. 10th edn. pp. 713-736 in Cox, F.E.G., Wakelin, D., Gillespie, S.H. \& Despommier, D.D. (Eds) Topley E Wilson's microbiology and microbial infections parasitology. London: Hodder Arnold.

Lewis, R., Behnke, J.M., Cassidy, J.P., Stafford, P., Murray, N. \& Holland, C.V. (2007) The migration of Ascaris suum larvae, and the associated pulmonary inflammatory response in susceptible C57BL/6j and resistant CBA/Ca mice. Parasitology 134, 1301-1314.

Lewis, R., Behnke, J.M., Stafford, P. \& Holland, C.V. (2006) The development of a mouse model to explore resistance and susceptibility to early Ascaris suum infection. Parasitology 132, 298-300.

Mercer, J.G., Mitchell, P.I., Moar, K.M., Bissett, A., Geissler, S., Bruce, K. \& Chappell, L.H. (2000) Anorexia in rats infected with the nematode, Nippostrongylus brasiliensis: experimental manipulations. Parasitology 120, 641-647.

O'Lorcain, P. \& Holland, C. (2000) The public health significance of Ascaris lumbricoides. Parasitology 121, S51-S71.

Ovington, K.S. (1985) Dose-dependent relationships between Nippostrongylus brasiliensis populations and rat food intake. Parasitology 91, 157-167.

Pawlowski, Z.S. \& Arfaa, F. (1985) Ascariasis.pp. 347-359 in Warren, K.S. \& Mahmoud, A.A.F. (Eds) Tropical and geographical medicine. New York: McGraw-Hill.

Roepstorff, A. \& Nansen, P. (1994) Epidemiology and control of helminth parasites in pigs under intensive and non-intensive production systems. Veterinary Parasitology 54, 69-85.

Stephenson, L.S. (1987) Ascariasis. In Impact of helminth infections on human nutrition, pp. 89-118 [L.S. Stephenson \& C.V. Holland, editors]. London: Taylor \& Francis.

Stephenson, L.S., Pond, W.G., Nesheim, M.C., Krook, L.P. \& Crompton, D.W.T. (1980) Ascaris suum: nutrient absorption, growth and intestinal pathology in young pigs experimentally infected with 15 day old larvae. Experimental Parasitology 49, 15-25.

Stewart, T.B., Bidner, T.D., Southern, L.L. \& Simmons, L.A. (1984) The efficacy of fenbendazole against migrating Ascaris suum larvae in pigs. American Journal of Veterinary Research 45, 984-986.

Hlaing, T. (1993) Ascariasis and childhood malnutrition. Parasitology 107, S125-S136.

WHO (2002) The world health report 2002, Geneva: World Health Organization pp. 186-192.

Yang, S., Gaafar, S.M. \& Bottoms, G.D. (1990) Serum levels of gastrin, insulin and glucagons as possible factors of anorexia in pigs infected once with Ascaris suum. Veterinary Parasitology 36, 211-219.

(Accepted 5 December 2007)

First published online 20 November 2008 (C) 2009 Cambridge University Press 